\title{
Review \\ Erythema Migrans-like COVID Vaccine Arm: A Literature Review
}

\author{
Gaia Fasano $^{1,+} \mathbb{D}$, Luigi Bennardo ${ }^{1, *,+} \mathbb{D}$, Silvana Ruffolo ${ }^{2}$, Maria Passante ${ }^{1}$, Azzurra Gaia Ambrosio ${ }^{1}$, \\ Maddalena Napolitano $^{3}{ }^{\mathbb{D}}$, Eugenio Provenzano ${ }^{2}$, Steven Paul Nisticò ${ }^{1}$ and Cataldo Patruno ${ }^{1}$
}

check for

updates

Citation: Fasano, G.; Bennardo, L.;

Ruffolo, S.; Passante, M.; Ambrosio,

A.G.; Napolitano, M.; Provenzano, E.;

Nisticò, S.P.; Patruno, C. Erythema

Migrans-like COVID Vaccine Arm: A

Literature Review. J. Clin. Med. 2022,

11, 797. https://doi.org/10.3390/

jcm11030797

Academic Editors: Gabriella

Fabbrocini, Claudio Marasca

and Stamatios Gregoriou

Received: 4 December 2021

Accepted: 30 January 2022

Published: 1 February 2022

Publisher's Note: MDPI stays neutral with regard to jurisdictional claims in published maps and institutional affiliations.

Copyright: (C) 2022 by the authors. Licensee MDPI, Basel, Switzerland. This article is an open access article distributed under the terms and conditions of the Creative Commons Attribution (CC BY) license (https:// creativecommons.org/licenses/by/ $4.0 /)$.
1 Department of Health Sciences, Magna Graecia University, 88100 Catanzaro, Italy; fasano.gaia@gmail.com (G.F.); mariapassante989@gmail.com (M.P.); azzurra.ambrosio@gmail.com (A.G.A.); steven.nistico@gmail.com (S.P.N.); cataldo.patruno@unicz.it (C.P.)

2 Mariano Santo Hospital, 87100 Cosenza, Italy; sruffolo@aocs.it (S.R.); eprovenzano0@gmail.com (E.P.)

3 Department of Health Sciences Vincenzo Tiberio, University of Molise, 86100 Campobasso, Italy; maddy.napolitano@gmail.com

* Correspondence: luigibennardo10@gmail.com

$\dagger$ These authors contributed equally to this work.

\begin{abstract}
COVID Vaccine Arm (CVA) is an adverse drug reaction from mRNA vaccine for SARS-CoV-2. CVA is characterized by erythema and edema on the vaccination site (usually deltoid area) that appears from 5 to 10 days after vaccination and is sometimes associated with itching or pain. The exact etiology of CVA is still unclear, but delayed hypersensitivity against an excipient seems to play an essential role in the pathogenesis of the disease. This work performs a systematic literature review on CVA using three different databases containing articles published until 10 November 2021. The literature review includes eight papers reporting single cases or case series of CVA. Moreover, it also addresses, other cutaneous reactions following COVID 19 vaccinations as well as possible differential diagnosis. CVA migrans-like erythema is characterized by a ring-shaped rash in the injection area, which appears some days after the injection and disappears in about 10 days. This reaction may appear more rapidly in subsequent doses.
\end{abstract}

Keywords: COVID Vaccine Arm (CVA); Spikevax ${ }^{\circledR}$ Moderna (mRNA-1273) vaccine; Cominarty ${ }^{\circledR}$ Pfizer/BioNTech's (BNT162b2) vaccine; COVID-19; Erythema migrans-like

\section{Introduction}

The Coronavirus disease-2019 (COVID-19) was officially declared pandemic by the WHO on 11 March 2020, and the rapid vaccine development became a global priority [1].

The Food and Drug Administration (FDA) in December 2020 authorized the use of Cominarty ${ }^{\circledR}$ Pfizer/BioNTech's (BNT162b2) and Spikevax ${ }^{\circledR}$ Moderna (mRNA-1273) COVID-19 vaccines in order to overcome the worldwide emergency [2].

COVID Vaccine Arm (CVA) is a recently observed transient skin reaction resulting from mRNA vaccination that affects approximately $2 \%$ of subjects who received the vaccine [3]. The emerging medical literature studying Moderna and Pfizer/BioNTech's vaccines described CVA [4].

CVA is characterized by erythema and edema at the vaccination site and appears from 5 to 10 days after vaccination.

CVA can also appear in different body parts, even if not close to the injection site [5]. Moreover, CVA is commonly associated with pain or burning sensation, but it can be asymptomatic [5-7].

Rarely, the CVA eruption has been mistakenly diagnosed as cellulitis and a systemic antibiotic therapy was recommended as prophylaxis, in addition to the topical corticosteroid therapy [3]. 
Usually, CVA resolves spontaneously within a few weeks. However, some patients are treated with systemic antihistamines, and topical or oral glucocorticoids in order to relieve subjective symptoms [8] (Figure 1).

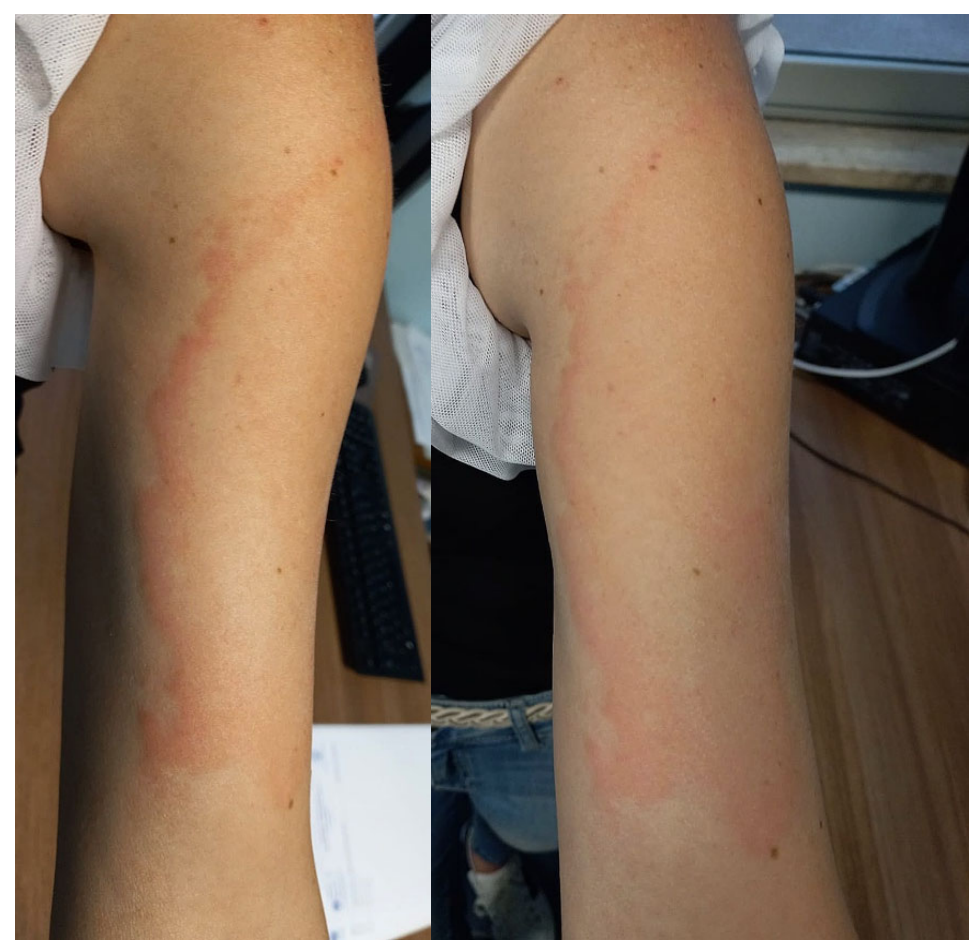

Figure 1. Erythematous, edematous, marginated Erythema migrans-like COVID Vaccine Arm eruption 7 days after the first dose of the Moderna vaccine.

The etiopathogenesis of CVA is still unclear and a delayed hypersensitivity reaction induced by some vaccine components is currently considered the most likely hypothesis [9].

The reaction after the first administration is not a contraindication to the second administration. However, patients and health care professionals should be aware that this type of reaction may develop more rapidly after the second vaccine dose $[10,11]$.

Given the high incidence of this reaction (up to $2 \%$ of vaccinated patients) and the relatively low number of reports, we performed a literature review about CVA, its differential diagnosis, and other cutaneous reactions caused by the COVID vaccine. The aim of the paper consists in improving the awareness of this reaction among clinicians and researchers.

\section{Materials and Methods}

The authors carried on a systematic literature review on CVA using the guidelines and the criteria established from the Preferred Reporting Items for Systematic Reviews and Meta-Analyses (PRISMA)

Two independent researchers (L.B. and G.F.) performed a comprehensive literature search to identify relevant studies from 20 July 2021 up to 10 November 2021, with no temporal restriction, using the following databases: MEDLINE/PubMed (National Center for Biotechnology Information, NCBI), EMBASE (Ovid), and Google Scholar. The search string contained Medical Subject Headings (MeSH) and free-text terms.

The research algorithm comprehended the following keywords: "COVID-19 vaccine arm", "skin COVID-19 vaccine", and "adverse skin reaction COVID-19 vaccine". We screened all articles' titles and abstracts containing such keywords.

In addition, we also searched for citations included in the reference list of the selected articles. After eliminating the duplicates, the eligible articles were screened based on the title and the abstract. Finally, we analyzed the full text of the articles potentially suitable for 
inclusion in the systematic reviews. In case of discrepancies among authors, a third senior researcher (C.P.) decided whether to include or not one article.

\section{Results}

The literature search identified 158 articles, 111 of which have been removed afterthe activity of screening of titles and abstracts. The full text of the remaining 47 papers was assessed for inclusion, and 2 articles were excluded as in non-english language; finally, 8 papers met the inclusion criteria; and, thus, they were included in the review. The article selection flow chart (Figure 2) summarizes the search strategy adopted in this study. We analyzed 5 case series and 3 case reports for a total of 29 patients.
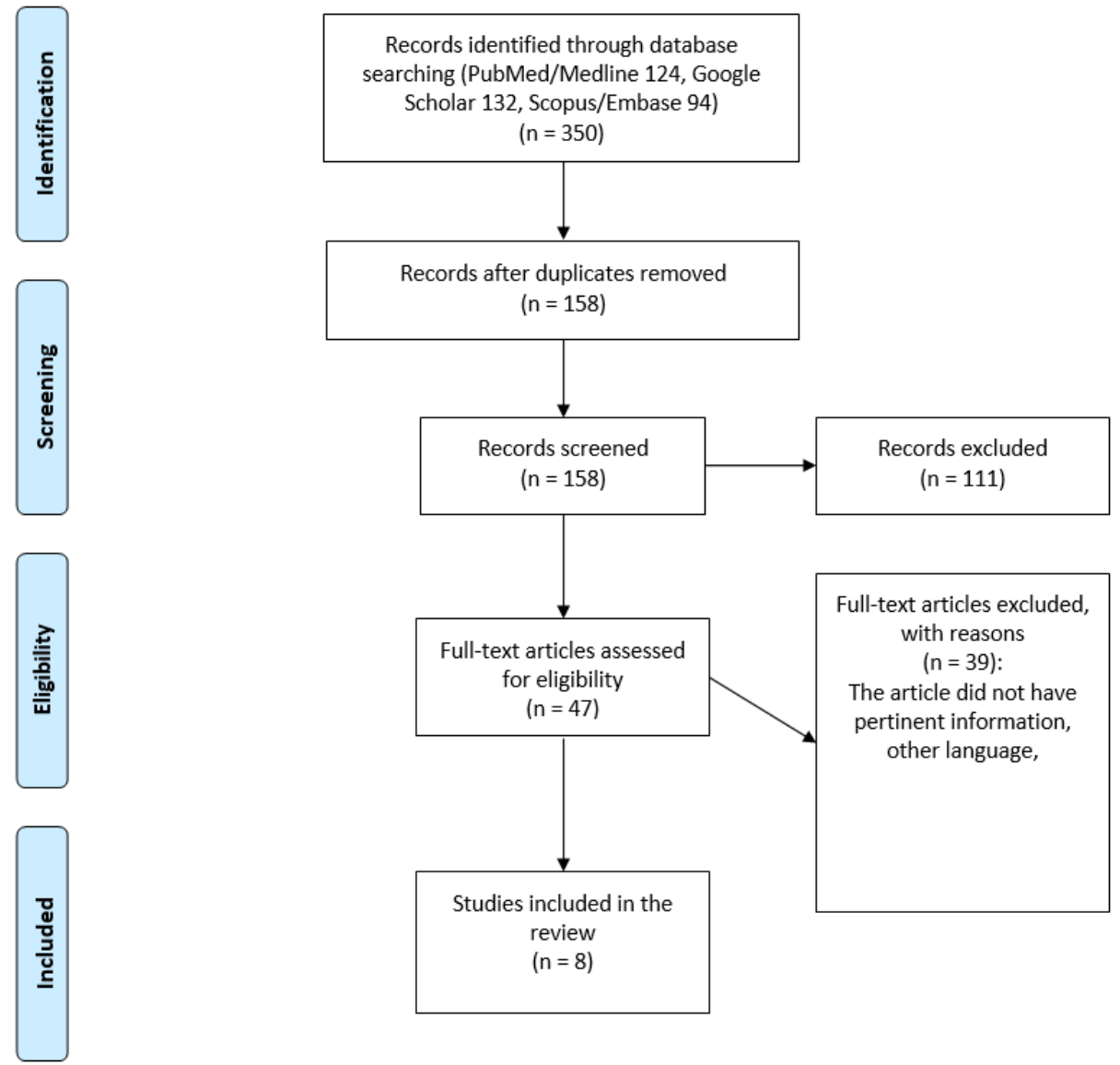

Figure 2. Articles selection flowchart.

All the patients reported a reaction near the injection site after the complete resolution of the other local and systemic symptoms associated with vaccination.

The reaction was reported both after Moderna vaccine (89.7\%) and Pfizer/BioNTech's vaccine $(10.3 \%)$ after first $(89.7 \%)$ or second dose $(10.3 \%)$ with a median onset on day 7.7 (range 3 to 11 ).

The median age (year) of patients was 56 (range 31 to 86 ), while $89.7 \%$ of patients were female.

In medical history, 9 patients (31\%) reported allergic predispositions such as pharmacological allergy, rhinitis, urticaria, angioedema, contrast allergy, wasp allergy, atopic history, and others. Moreover, patiens reported other sporadic comorbidities, probably not correlated to that reaction, such as psoriasis, atrial fibrillation, hypercholesterolemia, hypothyroidism, breast and ovarian cancer, melanoma, non-melanoma skin cancer, chronic obstructive pulmonary disease (COPD), and pulmonary hypertension.

Almost all patients reported erythema, red plaque, pruritus, warmth, swelling, scaling, and pain. 
Only $24.1 \%$ of patients reported systemic symptoms such as fever, headache, myalgia, chills, or other cutaneous symptoms not close to the injection area such as papules on the palm and fingers or urticarial plaques on the elbows. One patient reported lymphadenopathy, while another patient reported tachycardia and hypertension.

The rash diameter was detected only for 16 patients with a median of $9.9 \mathrm{~cm}$ (range 4 to 19.5).

Skin biopsy was performed only in 4 patients and reported a focal spongiosis with vacuolar alteration and few lymphocytes in the epidermis; an inflammatory perivascular infiltrate was found in the dermis with lymphocytes and some histocytes, eosinophilic granulocytes, and neutrophils.

Almost all patients did not undergo any therapy; some patients (41.4\%) used topical corticosteroids such as clobetasol propionate $0.05 \%$, mometasone furoate $0.01 \%$, methylprednisolone-aceponate $0.1 \%$, hydrocortisone $1 \%$, triamcinolone $0.1 \%$ or other topical agents such as diclofenac, diphenhydramine hydrochloride $1 \%$.

Only 7 patients $(24.1 \%$ ) used oral antihistamines such as cetirizine $10 \mathrm{mg}$, loratadine $10 \mathrm{mg}$, desloratadine, diphenhydramine $25 \mathrm{mg}$, diphenhydramine $25 \mathrm{mg}$, famotidine $20 \mathrm{mg}$.

The rash and the other cutaneous symptoms resolved spontaneously or thanks to the treatment about 4 days on average after the onset (range 1 to 7 ). Tables 1 and 2 report all the studies selected for this review as well as patients' characteristics.

Table 1. Selected studies.

\begin{tabular}{cccc}
\hline Author & Study Type & Number of Patients & Type of Vaccine \\
\hline Wei et al. [8] & Case series & 4 & Moderna mRNA-1273 \\
\hline Anshari et al. [9] & Case report & 1 & Moderna mRNA-1273 \\
\hline Zengarini et al. [12] & Case report & 1 & Moderna mRNA-1273 \\
\hline Kempf et al. [13] & Case series & 3 & Moderna mRNA-1273 \\
\hline Barriere et al. [11] & Case report & 1 & Cominarty (Pfizer/BioNTech) \\
\hline Gregoriou et al. [4] & Case series & 4 & $\begin{array}{c}\text { Moderna mRNA-1273 and } \\
\text { Cominarty (Pfizer/BioNTech) }\end{array}$ \\
\hline Blumenthal et al. [5] & Case series & 12 & $\begin{array}{c}\text { Moderna mRNA-1273 } \\
\text { Lindgren et al. [14] }\end{array}$ \\
\hline
\end{tabular}


Table 2. Characteristics of patients included in the review.

\begin{tabular}{|c|c|c|c|c|c|c|c|c|c|c|c|}
\hline $\begin{array}{l}\text { Study and Patient's } \\
\text { Number }\end{array}$ & Age & Gender & $\begin{array}{c}\text { Medical History } \\
\text { or Allergies }\end{array}$ & $\begin{array}{l}\text { Type of } \\
\text { Vaccine }\end{array}$ & $\begin{array}{c}\text { Days after } \\
\text { Vaccination }\end{array}$ & $\begin{array}{l}\text { Localized } \\
\text { Symptoms }\end{array}$ & $\begin{array}{c}\text { Rash } \\
\text { Diameter }\end{array}$ & $\begin{array}{c}\text { Systemic } \\
\text { Symptoms }\end{array}$ & Skin Biopsy & Therapy & Outcome \\
\hline Wei et al. [1] & 74 & Female & No & $\begin{array}{c}\text { Moderna } \\
\text { mRNA-1273 }\end{array}$ & $\begin{array}{l}8 \text { days after } \\
\text { first dose }\end{array}$ & $\begin{array}{l}\text { Pruritus, } \\
\text { erythematous } \\
\text { plaque, } \\
\text { mild scaling }\end{array}$ & $15 \mathrm{~cm}$ & No & No & $\begin{array}{c}\text { Topical clobetasol } \\
\text { proprionate } 0.05 \% \mathrm{cream} \\
\text { and oral cetirizine } 10 \mathrm{mg}\end{array}$ & $\begin{array}{l}\text { Partial } \\
\text { resolution } \\
\text { after 1 week }\end{array}$ \\
\hline Wei et al. [2] & 62 & Female & No & $\begin{array}{c}\text { Moderna } \\
\text { mRNA-1273 }\end{array}$ & $\begin{array}{l}8 \text { days after } \\
\text { first dose }\end{array}$ & $\begin{array}{c}\text { Pruritus, } \\
\text { erythematous } \\
\text { plaque, } \\
\text { edema, warmth }\end{array}$ & Not reported & No & No & $\begin{array}{c}\text { Mometasone furoate } \\
0.01 \% \text { ointment, } \\
\text { diphenhydramine } \\
\text { hydrochloride } 1 \% \text { cream, } \\
\text { oral loratadine } 10 \mathrm{mg}\end{array}$ & \\
\hline Wei et al. [3] & 54 & Female & No & $\begin{array}{c}\text { Moderna } \\
\text { mRNA-1273 }\end{array}$ & $\begin{array}{l}7 \text { days after } \\
\text { first dose }\end{array}$ & $\begin{array}{l}\text { Erythematous } \\
\text { rash }\end{array}$ & Not reported & No & No & No & $\begin{array}{l}\text { Resolving } \\
\text { sponta- } \\
\text { neously in } \\
4 \text { days }\end{array}$ \\
\hline Wei et al. [4] & 72 & Female & $\begin{array}{c}\text { Psoriasis, } \\
\text { atrial fibrillation, } \\
\text { hypercholes- } \\
\text { terolemia, } \\
\text { hypothyroidism }\end{array}$ & $\begin{array}{c}\text { Moderna } \\
\text { mRNA-1273 }\end{array}$ & $\begin{array}{l}10 \text { days after } \\
\text { first dose }\end{array}$ & $\begin{array}{c}\text { Pruritus, } \\
\text { Erythematous } \\
\text { plaque, warmth }\end{array}$ & $14 \mathrm{~cm}$ & No & No & No & $\begin{array}{l}\text { Resolving } \\
\text { sponta- } \\
\text { neously in } \\
2 \text { days }\end{array}$ \\
\hline Anshari et al. [1] & 56 & Female & $\begin{array}{l}\text { Breast cancer, } \\
\text { atopic history } \\
\text { with eczema and } \\
\text { allergic rhinitis, } \\
\text { thalassemia trait }\end{array}$ & $\begin{array}{c}\text { Moderna } \\
\text { mRNA-1273 }\end{array}$ & $\begin{array}{l}3 \text { days after } \\
\text { second dose }\end{array}$ & $\begin{array}{l}\text { Swell, redness, } \\
\text { warm, pain }\end{array}$ & Not reported & No & $\begin{array}{l}\text { Focal spongiosis and vacuolar } \\
\text { alteration in the epidermidis. } \\
\text { Inflammatory infiltrate } \\
\text { perivascular in the dermis with } \\
\text { lymphocytes and some } \\
\text { histocytes and neutrophils. }\end{array}$ & $\begin{array}{l}\text { Surface cooling } \\
\text { and compression }\end{array}$ & $\begin{array}{l}\text { Resolution } \\
\text { after } 5 \text { days }\end{array}$ \\
\hline Zengarini et al. [1] & 63 & Female & No & $\begin{array}{l}\text { Moderna } \\
\text { mRNA-1273 }\end{array}$ & $\begin{array}{l}5 \text { days after } \\
\text { first dose }\end{array}$ & $\begin{array}{c}\text { Flat and } \\
\text { targetoid } \\
\text { erythema with } \\
\text { bull's } \\
\text { eye aspect }\end{array}$ & Not reported & No & No & $\begin{array}{l}\text { Surface cooling } \\
\text { and compression }\end{array}$ & $\begin{array}{l}\text { Resolution } \\
\text { after } 2 \text { days }\end{array}$ \\
\hline Kempf et al. [1] & 84 & Male & $\begin{array}{l}\text { Melanoma, } \\
\text { non-melanoma } \\
\text { skin cancer }\end{array}$ & $\begin{array}{c}\text { Moderna } \\
\text { mRNA-1273 }\end{array}$ & $\begin{array}{l}7 \text { days after } \\
\text { first dose }\end{array}$ & Erythema & Not reported & No & $\begin{array}{l}\text { Focal spongiosis and } \\
\text { exocytosis of a few } \\
\text { lymphocytes in the } \\
\text { epidermidis. Inflammatory } \\
\text { infiltrate perivascular in the } \\
\text { dermis with lymphocytes and } \\
\text { some eosinophilic granulocytes } \\
\text { and neutrophils. }\end{array}$ & & \\
\hline Kempf et al. [2] & 86 & Female & $\begin{array}{l}\text { Non-melanoma } \\
\text { skin cancer }\end{array}$ & $\begin{array}{c}\text { Moderna } \\
\text { mRNA-1273 }\end{array}$ & $\begin{array}{l}7 \text { days after } \\
\text { first dose }\end{array}$ & Erythema & Not reported & No & $\begin{array}{l}\text { Focal spongiosis and } \\
\text { exocytosis of a few } \\
\text { lymphocytes in the } \\
\text { epidermidis. Inflammatory } \\
\text { infiltrate perivascular in the } \\
\text { dermis with lymphocytes and } \\
\text { some eosinophilic granulocytes } \\
\text { and neutrophils. }\end{array}$ & & \\
\hline
\end{tabular}


Table 2 Cont.

\begin{tabular}{|c|c|c|c|c|c|c|c|c|c|c|c|}
\hline $\begin{array}{l}\text { Study and Patient's } \\
\text { Number }\end{array}$ & Age & Gender & $\begin{array}{c}\text { Medical History } \\
\text { or Allergies }\end{array}$ & $\begin{array}{l}\text { Type of } \\
\text { Vaccine }\end{array}$ & $\begin{array}{c}\text { Days after } \\
\text { Vaccination }\end{array}$ & $\begin{array}{l}\text { Localized } \\
\text { Symptoms }\end{array}$ & $\begin{array}{c}\text { Rash } \\
\text { Diameter }\end{array}$ & $\begin{array}{c}\text { Systemic } \\
\text { Symptoms }\end{array}$ & Skin Biopsy & Therapy & Outcome \\
\hline Kempf et al. [3] & 81 & Female & $\begin{array}{l}\text { Non-melanoma } \\
\text { skin cancer, } \\
\text { eczema of hand }\end{array}$ & $\begin{array}{c}\text { Moderna } \\
\text { mRNA-1273 }\end{array}$ & $\begin{array}{l}7 \text { days after } \\
\text { first dose }\end{array}$ & Erythema & Not reported & $\begin{aligned} \\
\mathrm{i} \\
\mathrm{d} \\
\text { so }\end{aligned}$ & $\begin{array}{l}\text { Focal spongiosis and } \\
\text { exocytosis of a few } \\
\text { lymphocytes in the } \\
\text { epidermidis. Inflammatory } \\
\text { infiltrate perivascular in the } \\
\text { dermis with lymphocytes and } \\
\text { some eosinophilic granulocytes } \\
\text { and neutrophils. }\end{array}$ & & \\
\hline Barriere et al. [1] & 76 & Female & $\begin{array}{l}\text { Ovarian } \\
\text { Neoplasia }\end{array}$ & $\begin{array}{l}\text { Comirnaty } \\
\text { Pfizer- } \\
\text { Biontech }\end{array}$ & $\begin{array}{l}5 \text { days } \\
\text { after the } \\
\text { second dose }\end{array}$ & $\begin{array}{l}\text { Inflammatory } \\
\text { edema, pain }\end{array}$ & Not reported & No & No & & \\
\hline Gregoriou et al. [1] & 733 & Female & No & $\begin{array}{c}\text { Moderna } \\
\text { mRNA-1273 }\end{array}$ & $\begin{array}{l}9 \text { days after } \\
\text { first dose }\end{array}$ & $\begin{array}{l}\text { Erythematous } \\
\text { papules with } \\
\text { red plaque, } \\
\text { scaling, pruritus }\end{array}$ & $7 \mathrm{~cm}$ & No & No & $\begin{array}{c}\text { Topical } \\
\text { methylprednisolone- } \\
\text { aceponate } 0.1 \% \text { cream } \\
\text { and desloratadine }\end{array}$ & $\begin{array}{l}\text { Resolution } \\
\text { after } 4 \text { days }\end{array}$ \\
\hline Gregoriou et al. [2] & 74 & Female & $\begin{array}{c}\text { Chronic } \\
\text { obstructive } \\
\text { pulmonary } \\
\text { disease (COPD), } \\
\text { pulmonary } \\
\text { hypertension }\end{array}$ & $\begin{array}{l}\text { Cominarty } \\
\text { (Pfizer / } \\
\text { BioNTech) }\end{array}$ & $\begin{array}{l}8 \text { days after } \\
\text { second dose }\end{array}$ & $\begin{array}{l}\text { Erythematous } \\
\text { plaque }\end{array}$ & Not reported & No & No & $\begin{array}{c}\text { Topical } \\
\text { methylprednisolone- } \\
\text { aceponate } \\
0.1 \% \text { cream }\end{array}$ & $\begin{array}{l}\text { Resolution } \\
\text { after } 3 \text { days }\end{array}$ \\
\hline Gregoriou et al. [3] & 51 & Female & No & $\begin{array}{c}\text { Moderna } \\
\text { mRNA-1273 }\end{array}$ & $\begin{array}{l}9 \text { days after } \\
\text { first dose }\end{array}$ & $\begin{array}{l}\text { Erythematous } \\
\text { plaque }\end{array}$ & Not reported & No & No & $\begin{array}{c}\text { Topical } \\
\text { methylprednisolone- } \\
\text { aceponate } \\
0.1 \% \text { cream }\end{array}$ & $\begin{array}{l}\text { Resolution } \\
\text { after } 3 \text { days }\end{array}$ \\
\hline Gregoriou et al. [4] & 53 & Female & No & $\begin{array}{c}\text { Moderna } \\
\text { mRNA-1273 }\end{array}$ & $\begin{array}{l}11 \text { days after } \\
\text { first dose }\end{array}$ & $\begin{array}{l}\text { Erythematous } \\
\text { plaque }\end{array}$ & $8 \mathrm{~cm}$ & No & No & $\begin{array}{l}\text { Topical mometasone } \\
\text { furoate } 0.1 \% \text { cream }\end{array}$ & $\begin{array}{l}\text { Resolution } \\
\text { after } 5 \text { days }\end{array}$ \\
\hline Blumenthal et al. [1] & 37 & Female & No & $\begin{array}{c}\text { Moderna } \\
\text { mRNA-1273 }\end{array}$ & $\begin{array}{l}8 \text { days after } \\
\text { first dose }\end{array}$ & $\begin{array}{l}\text { Annular } \\
\text { papules, } \\
\text { pruritus }\end{array}$ & $9 \mathrm{~cm}$ & No & No & No & \\
\hline Blumenthal et al. [2] & 61 & Female & Contrast allergy & $\begin{array}{c}\text { Moderna } \\
\text { mRNA-1273 }\end{array}$ & $\begin{array}{l}8 \text { days after } \\
\text { first dose }\end{array}$ & $\begin{array}{c}\text { Edematous } \\
\text { plaque, } \\
\text { pruritus, } \\
\text { warmth }\end{array}$ & $10 \mathrm{~cm}$ & No & No & $\begin{array}{c}\text { Topical clobetasol } \\
\text { propionate } 0.05 \% \text { cream }\end{array}$ & \\
\hline Blumenthal et al. [3] & 45 & Female & $\begin{array}{l}\text { Rhinits, } \\
\text { penicillin allergy }\end{array}$ & $\begin{array}{c}\text { Moderna } \\
\text { mRNA-1273 }\end{array}$ & $\begin{array}{l}8 \text { days after } \\
\text { first dose }\end{array}$ & $\begin{array}{l}\text { Edematous } \\
\text { plaque, } \\
\text { pruritus, pain }\end{array}$ & $14 \mathrm{~cm}$ & $\begin{array}{l}\text { Fatigue, } \\
\text { headache, } \\
\text { myalgias, } \\
\text { chills }\end{array}$ & No & $\begin{array}{c}\text { Topical hydrocortisone } \\
1 \% \text { cream, } \\
\text { diphenhydramine } 25 \mathrm{mg}\end{array}$ & \\
\hline Blumenthal et al. [4] & 31 & Female & Urticaria, rhinits & $\begin{array}{c}\text { Moderna } \\
\text { mRNA-1273 }\end{array}$ & $\begin{array}{l}8 \text { days after } \\
\text { first dose }\end{array}$ & $\begin{array}{l}\text { Erythematous } \\
\text { plaque, } \\
\text { pruritus }\end{array}$ & $5 \mathrm{~cm}$ & Lymphadenopathy & No & $\begin{array}{c}\text { Topical triamcinolone } \\
0.1 \% \text { cream, diclofenac } \\
1 \% \text { topical gel, cetirizine } \\
10 \mathrm{mg}\end{array}$ & \\
\hline
\end{tabular}


Table 2 Cont.

\begin{tabular}{|c|c|c|c|c|c|c|c|c|c|c|c|}
\hline $\begin{array}{c}\text { Study and Patient's } \\
\text { Number }\end{array}$ & Age & Gender & $\begin{array}{c}\text { Medical History } \\
\text { or Allergies }\end{array}$ & $\begin{array}{l}\text { Type of } \\
\text { Vaccine }\end{array}$ & $\begin{array}{c}\text { Days after } \\
\text { Vaccination }\end{array}$ & $\begin{array}{l}\text { Localized } \\
\text { Symptoms }\end{array}$ & $\begin{array}{c}\text { Rash } \\
\text { Diameter }\end{array}$ & $\begin{array}{c}\text { Systemic } \\
\text { Symptoms }\end{array}$ & Skin Biopsy & Therapy & Outcome \\
\hline Blumenthal et al. [5] & 40 & Female & No & $\begin{array}{c}\text { Moderna } \\
\text { mRNA-1273 }\end{array}$ & $\begin{array}{l}4 \text { days after } \\
\text { first dose }\end{array}$ & $\begin{array}{l}\text { Erythematous } \\
\text { plaque, } \\
\text { pruritus, pain }\end{array}$ & $13 \mathrm{~cm}$ & $\begin{array}{l}\text { Papules on } \\
\text { the palm and } \\
\text { fingers. } \\
\text { Headache, } \\
\text { fatigue, fever }\end{array}$ & No & No & \\
\hline Blumenthal et al. [6] & 43 & Male & No & $\begin{array}{c}\text { Moderna } \\
\text { mRNA-1273 }\end{array}$ & $\begin{array}{l}9 \text { days after } \\
\text { first dose }\end{array}$ & $\begin{array}{l}\text { Erythematous } \\
\text { plaque, } \\
\text { pruritus, pain, } \\
\text { warmth }\end{array}$ & $12.5 \mathrm{~cm}$ & $\begin{array}{l}\text { Urticarial } \\
\text { plaques on } \\
\text { the elbows }\end{array}$ & No & $\begin{array}{c}\text { Diphenhydramine } \\
25 \mathrm{mg} \text {, famotidine } 20 \mathrm{mg}\end{array}$ & \\
\hline Blumenthal et al. [7] & 38 & Female & Wasp allergy & $\begin{array}{c}\text { Moderna } \\
\text { mRNA-1273 }\end{array}$ & $\begin{array}{l}9 \text { days after } \\
\text { first dose }\end{array}$ & $\begin{array}{l}\text { Erythematous } \\
\text { plaque, pain }\end{array}$ & $7 \mathrm{~cm}$ & No & No & Loratadine $10 \mathrm{mg}$ & \\
\hline Blumenthal et al. [8] & 49 & Female & $\begin{array}{l}\text { Idiopathic } \\
\text { urticaria }\end{array}$ & $\begin{array}{c}\text { Moderna } \\
\text { mRNA-1273 }\end{array}$ & $\begin{array}{l}8 \text { days after } \\
\text { first dose }\end{array}$ & $\begin{array}{c}\text { Indurated } \\
\text { plaque, } \\
\text { pruritus, pain, } \\
\text { burning, } \\
\text { warmth }\end{array}$ & $4 \mathrm{~cm}$ & No & No & No & \\
\hline Blumenthal et al. [9] & 41 & Female & No & $\begin{array}{l}\text { Moderna } \\
\text { mRNA-1273 }\end{array}$ & $\begin{array}{l}10 \text { days after } \\
\text { first dose }\end{array}$ & $\begin{array}{c}\text { Indurated } \\
\text { plaque, } \\
\text { pruritus, } \\
\text { warmth } \\
\end{array}$ & $7.5 \mathrm{~cm}$ & Fatigue & No & No & \\
\hline Blumenthal et al. [10] & 47 & Male & $\begin{array}{c}\text { Almond allergy, } \\
\text { rhinits }\end{array}$ & $\begin{array}{c}\text { Moderna } \\
\text { mRNA-1273 }\end{array}$ & $\begin{array}{l}11 \text { days after } \\
\text { first dose }\end{array}$ & $\begin{array}{l}\text { Erythematous } \\
\text { plaque, pain }\end{array}$ & $7 \mathrm{~cm}$ & $\begin{array}{l}\text { Fatigue, } \\
\text { myalgias }\end{array}$ & No & No & \\
\hline Blumenthal et al. [12] & 46 & Female & Penicillin allergy & $\begin{array}{l}\text { Moderna } \\
\text { mRNA-1273 }\end{array}$ & $\begin{array}{l}9 \text { days after } \\
\text { first dose }\end{array}$ & $\begin{array}{c}\text { Erythematous } \\
\text { plaque, } \\
\text { pruritus }\end{array}$ & $7 \mathrm{~cm}$ & Headache & No & No & \\
\hline Lindgren et al. [1] & 60 & Female & No & $\begin{array}{l}\text { Moderna } \\
\text { mRNA-1273 }\end{array}$ & $\begin{array}{l}6 \text { days after } \\
\text { first dose }\end{array}$ & $\begin{array}{c}\text { Erythematous } \\
\text { papules with } \\
\text { pruritus, } \\
\text { swollen, pain }\end{array}$ & Not reported & No & No & $\begin{array}{l}\text { Topical clobetasol } \\
0.05 \% \text { cream }\end{array}$ & $\begin{array}{l}\text { Resolution } \\
\text { after } 1 \text { day }\end{array}$ \\
\hline Lindgren et al. [2] & 44 & Female & No & $\begin{array}{l}\text { Cominarty } \\
\text { (Pfizer/ } \\
\text { BioNTech) }\end{array}$ & $\begin{array}{l}7 \text { days after } \\
\text { first dose }\end{array}$ & $\begin{array}{l}\text { Erythema, pain, } \\
\text { pruritus, } \\
\text { sweeling }\end{array}$ & Not reported & $\begin{array}{l}\text { Fever, chills, } \\
\text { headache, } \\
\text { myalgias }\end{array}$ & No & $\begin{array}{l}\text { Topical triamcinolone } \\
0.1 \% \text { cream }\end{array}$ & $\begin{array}{l}\text { Resolution } \\
\text { after } 2 \text { days }\end{array}$ \\
\hline Lindgren et al. [3] & 33 & Female & No & $\begin{array}{l}\text { Moderna } \\
\text { mRNA-1273 }\end{array}$ & $\begin{array}{l}7 \text { days after } \\
\text { first dose }\end{array}$ & $\begin{array}{l}\text { Erythema, pain, } \\
\text { pruritus, } \\
\text { swelling }\end{array}$ & Not reported & No & No & $\begin{array}{c}\text { Topical hydrocortisone } \\
1 \% \text { cream }\end{array}$ & $\begin{array}{l}\text { Resolution } \\
\text { after } 4 \text { days }\end{array}$ \\
\hline
\end{tabular}




\section{Discussion}

The first 4 cases of CVA after the first dose of Moderna vaccine have been described by Wei et al. [8].

Anshari et al. [9] evidenced the case of a 56-old woman who manifested CVA 3 days after booster vaccination using Moderna vaccine. The symptoms disappeared with surface cooling and compression stocking 8 days later. Histological examination showed spongiosis with vacuolar alterations and perivascular inflammatory infiltrate in periadnexal areas, on superficial and deep dermal plexi, and subcutaneous fat, consisting of lymphocytes and some histiocytes with few intravascular neutrophils. Eosinophils were not present as signs of vascular wall damage. The biopsy result was consistent with delayed-type hypersensitivity.

Zengarini et al. [12] reported the case of a 63-years-old female who presented flat and targetoid erythema without other local symptoms. This manifestation was suspected to be an erythema migrans due to the marginate clinical aspect and to the fact that the patient was from an endemic area for Borrelia burgdorferi. The patient was vaccinated using a first dose of the Moderna vaccine 5 days prior to the appearance of the rash. The rash did not appear on the vaccine injection site, but in a different body area. In literature, Blumenthal et al. described delayed large local reactions even far from the injection site [5]. These reactions appeared on the same arm where the vaccine was injected, but away from the puncture site [5]. Wei et al. described it as "COVID vaccine arm" because it showed up some days after the first dose of vaccine [8]. COVID vaccine arm is similar to the insect bites' reactions. However, it can be distinguised through clinical history [15].

Kempf et al. [13] analyzed skin biopsy of 3 patients who had erythema on the left arm 6-7 days after the first dose of Moderna vaccine. Histology revealed epidermal changes with spongiosis and exocytosis of a few lymphocytes. Small lymphocytes (CD4+ and CD8+) and eosinophilic granulocytes have been reported in the dermal perivascular inflammatory infiltrate. The immunophenotypic profile revealed the presence of CD3+ T cells, CD4+ T cells, Tregs, plasma cells, and PDCs. Eosinophils were present in a variable number. The Authors concluded that the COVID vaccine arm has an immunological pattern which can be interpreted as a delayed-type hypersensitivity reaction [13].

Barriere et al. [11] described the case of a 76-year-old female with ovarian neoplasia who developed oedema without erythema, pain, and a $2 \mathrm{~cm}$ painless axillary lymphadenopathy, 5 days after the second dose of Pfizer/BioNTech's vaccine. PET/FDG imaging showed a complete metabolic response to the peritoneal target, but hypermetabolism in the lymph node and in the deltoid muscle was found [11]. Vaccination is the cause of a transient locoregional inflammatory reaction with inflammation of lymph nodes that can induce positive findings on FDG-PET [11]. Is important to know this local reaction to avoid invasive diagnostic and therapeutic procedures [11].

Gregoriou et al. described 4 cases of CVA, one of which was after Pfizer/BioNTech's vaccine. [4].

In healthcare workers, D. Fernandez-Nieto et al. [3] analyzed the skin manifestation of the ${ }^{\circledR}$ Pfizer/BioNTech vaccine. The researchers analyzed 4775 subjects who received the Pfizer/BioNTech vaccination; $18 \%$ of them experienced general side effects. $2 \%$ (103 people) had delayed skin reactions: $47.6 \%$ (49/193) after the first vaccine dose, 52.4\% (54/103) after the second dose. $32.7 \%(16 / 49)$ had recurrance after the second dose. The reaction duration was variable: in $22.3 \%$ of patients, the reaction resolved in less than $8 \mathrm{~h}$; in $26.2 \%$ of patients the reaction lasted between 8 and $24 \mathrm{~h}$; in another $36.9 \%$ of workers it lasted between 48 and $72 \mathrm{~h}$ and only in $13.6 \%$ of cases it lasted more than $72 \mathrm{~h} .68 \%$ of patients experienced itch (70 patients); $4.9 \%$ presented local or disseminated reactions (5 patients). None of the patients developed an anaphylactic reaction. A skin biopsy with histological examination was performed on a patients who presented an erythematous targetoid patch on the injection site. The biopsy showed a superficial and deep perivascular lymphocytic infiltrate with dilated vessels and intraluminal neutrophils. Immunohistochemistry for the SARS-CoV-2 spike 1A9 protein was negative. 
Recently, a case series of delayed large local reactions to the Moderna vaccine [5], including 12 cases, showed that the average onset of reaction after the first dose was about day 8 and the resolution average time was 6 days. Six patients experienced similar reactions also after the second dose with an average onset of 2 days. The reaction onset after the second dose was earlier with respect to the first dose [5]. Another study showed that patients who experienced CVA-both after the first and second vaccine dose-experienced symptoms faster after the second than the first injection (1-3 days) [16].

\subsection{Cutaneous Reactions after COVID-19 Vaccination}

Both COVID-19 infection and COVID-19 vaccines can cause multiple cutaneous reactions [17].

In clinical trials of 11 authorized COVID-19 vaccines, the most common adverse cutaneous reactions were local injection site reactions: erythema, pain, itching, swelling, pruritus, and tension on the injection site; the symptoms resolved over the next 24 to $48 \mathrm{~h}$ [18]. In phase III of Moderna clinical trials, among 15,185 participants who got vaccination 228 (1.5\%) developed, within 7 days after the first dose, delayed large local reactions such as erythema, induration, and tenderness [19]. After the second dose, $68(0.2 \%)$ of participants developed delayed large local reactions [19]. Less common observed reactions are: allergic, atopic and contact dermatitis, eczema, exfoliative rash, and vesicular rash [19].

Urticaria, angio-oedema, and anaphylaxis are type I hypersensitivity reactions due to allergy to some ingredients; they are not very common although theycan be severe [17].

Another type of reaction induced by Moderna vaccine is the erythema multiforme [20-22]. Muhamad Khalid et al. [23] reported the case of a patient who developed a mild rash 2 weeks after the first dose that resolved without any treatment. After the second dose, the patient developed large blisters and redness to the anterior chest, genitalia, bilateral hands, and bilateral lower feet without facial or mucosal involvement. There were no other associated systemic symptoms. The biopsy showed the presence of eosinophils that were suggestive of drug-induced erythema multiforme. Likely, the cause of it is due to the temporal relationship between vaccination and rash development.

Ackerman $\mathrm{M}$ et al. [24] described a morbilliform rash (maculopapular, pruritic exanthem) that erupted over 30\% of a patient's body. The rash developed on the face, trunk, upper extremities, sparing oral and genital mucosa, followed by a systemic manifestation with liver injury. Rash and liver damage enzymes improved after corticosteroid treatment.

Type IV hypersensitivity reactions on previous radiation sites are also described [17].

Soyfer et al. [25] described a dermatitis in previously irradiated skin sites of 2 patients after Pfizer/BioNTech's vaccination.

Delayed inflammatory reactions in the site of dermal hyaluronic acid fillers have been described; Munavalli et al. [26] described fifteen cases: 11 after Moderna vaccine and 4 after Pfizer/BioNTech's vaccine. The areas treated with fillers showed up swelling and inflammation 24-48 $\mathrm{h}$ after the vaccination. These reactions have also been observed after other vaccines, such as influence and in patients with COVID-19 infection [17]. Ethiopathological mechanism is probably related to the expression of angiotensin-converting enzyme (ACE) receptors in adipose tissue where the fillers have been injected [26].

The vaccine-induced spike protein would determine the stimulation of ACE2 which is its target. This would induce stimulation of CD8 in particular and subsequent Th1 inflammatory response. This mechanism could be confirmed by similar reactions observed in the granuloma from vaccination against TB with BCG in some healthcare professionals, always after administration of the two mRNA vaccines [27]. ACE blockers is the therapy to prefer over the corticosteroid, as the latter can reduce vaccine efficacy [17].

Lopatynsky-Reyes et al. [27] described a local skin inflammation in scar sites due to previous BCG vaccination accompanied by headache, myalgia, malaise, and arthralgia one day following the second dose of both Pfizer/BioNTech's and Moderna vaccination.

Chilblain-like lesions have been observed after both COVID-19 infections and the COVID-19 vaccine. These lesions appear as erythematous, violaceous papules, and 
macules on the hands and feet; treatment with topical steroids can reduce the symptoms [18]. Qisi et al. [18] described 10 cases of pernio and chilblains: 6 associated with Pfizer/BioNTech's vaccine and 4 associated with Moderna vaccine.

Lichen planus, herpes simplex, reactivation of herpes zoster, pityriasis rosea (reactivation of HHV 6 and HHV 7), maculopapular rash, swelling of the face, erythromelalgia, and petechial rash were very rare. No severe adverse reactions were reported [18,28-30].

Leukocytoclastic vasculitis, lupus erythematosus, and immune thrombocytopenia are possible immuno-mediated skin reactions reported [17].

\subsection{Differential Diagnosis}

CVA must be differentiated from other skin eruptions.

CVA associated with systemic symptoms has been misdiagnosed for cellulitis [14]. It is possible to distinguish CVA from cellulitis based on the time of the onset (1 week vs. 5 days), absence of systemic symptoms, resolution time of approximately $4-5$ days, spontaneous resolution, or rapid response to treatment with topical corticosteroids and antihistamines. Pruritus is also commonly found in CVA manifestations. [9,14].

Halperin, et al. [12] defined the diagnostic criteria to differentiate between cellulitis and local reaction post-vaccination. Cellulitis had three main symptoms: local pain, erythema, induration/swelling, and warmth [9]. Furthermore, the response to antibiotics can help confirm the diagnosis [9].

Montjoye et al. [31] described the case of eosinophilic cellulitis or Wells syndrome after Pfizer/BioNTech's vaccine. A 71-year-old woman presented on the right arm a painful eruption the day after the second dose. After 12 days, this eruption became erythematous and swollen with vesciculobullous lesions and erosions without fever. The suspecting cellulitis, a combination of amoxicillin and clavulanic acid was administered. Blood tests showed hypereosinophilia and slightly elevated C-reactive protein levels. Histological examination showed spongiotic dermatitis with dermal infiltrate (lymphocytes, histiocytes and eosinophils) and epidermal vesicles.A diagnosis of eosinophilic cellulitis was made. Vaccination is probably a triggering factor for this hypersensitivity reaction [31].

Ashley et al. [32] reported pediatric eosinophilic cellulitis 10 days after receiving tetanus, diphtheria, pertussis, and polio and measles, mumps, rubella, and varicella vaccines. The patch test showed a 1+ reaction to aluminum, hydroxide and neomycin at $96 \mathrm{~h}$, [32]. HoweverPfizer/BioNTech's vaccine does not contain these components, and further studies are needed to investigate the underlying etiopathogenetic cause.

We can distinguish CVA from erythema migrans through the absence of: recent tick bites, systemic neurological symptoms, IgM and IgG anti-Borrelia, and the rapid response to treatment with topical corticosteroids and antihistamines [12].

\section{Conclusions}

Vaccines are powerful and essential weapons against COVID-19 emergency. Skin side effects are generally minor and self-limited and should not discourage vaccination.

CVA is a mild possible side effect and not a contraindication for the second dose [8]. However, patients and health care should be aware that this reaction may develop more rapidly after the second dose [10].

CVA migrans-like erythema is an adverse reaction characterized by a ring erythematous rash in the injection area, which occurs some days after the vaccine and resolves in about 10 days [5]. CVA should be distinguished from the more common local reactions which are observed one day after vaccination and which last 2 to 3 days [33]. It is essential to know the possible heterogeneity of CVA and educate the general practitioners to recognize, through a detailed analysis of family and personal history, all the various manifestations associated with the Moderna vaccine. 
Author Contributions: Conceptualization, L.B. and G.F.; methodology, A.G.A.; software, M.P.; validation, C.P., E.P. and S.P.N.; formal analysis, S.R.; data curation, M.N.; writing-original draft preparation, G.F.; writing-review and editing, C.P. and L.B.; supervision, S.P.N. All authors have read and agreed to the published version of the manuscript.

Funding: This research received no external funding.

Institutional Review Board Statement: The study was conducted according to the guidelines of the Declaration of Helsinki. Ethical review and approval were waived for this study, due to the type of study (Case report).

Informed Consent Statement: Informed consent was obtained from all subjects involved in the study. Written informed consent has been obtained from the patient to publish this paper.

Data Availability Statement: The data presented in this study are available on request from the corresponding author. The data are not publicly available due to privacy.

Conflicts of Interest: The authors declare no conflict of interest.

\section{References}

1. Jin, Y.; Yang, H.; Ji, W.; Wu, W.; Chen, S.; Zhang, W.; Duan, G. Virology, epidemiology, pathogenesis, and control of COVID-19. Viruses 2020, 12, 372. [CrossRef]

2. Centers for Disease Control and Prevention. COVID Data Tracker. Available online: http://covid.cdc.gov/covid-data-tracker/ \#global-counts-rates (accessed on 12 February 2021).

3. Fernandez-Nieto, D.; Hammerle, J.; Fernandez-Escribano, M.; Moreno-Del Real, C.M.; Garcia-Abellas, P.; Carretero-Barrio, I.; Solano-Solares, E.; de-la-Hoz-Caballer, B.; Jimenez-Cauhe, J.; Ortega-Quijano, D.; et al. Skin manifestations of the BNT162b2 mRNA COVID-19 vaccine in healthcare workers. 'COVID-arm': A clinical and histological characterization. J. Eur. Acad. Dermatol. Venereol. 2021, 35, e425-e427. [CrossRef] [PubMed]

4. Gregoriou, S.; Kleidona, I.A.; Tsimpidakis, A.; Nicolaidou, E.; Stratigos, A.; Rigopoulos, D. 'COVID vaccine arm' may present after both mRNA vaccines vaccination. J. Eur. Acad. Dermatol. Venereol. 2021, 35, e867-e868. [CrossRef] [PubMed]

5. Blumenthal, K.G.; Freeman, E.E.; Saff, R.R. Delayed large local reactions to mRNA-1273 vaccine against SARS-CoV-2. N. Engl. J. Med. 2021, 384, 1273-1277. [CrossRef] [PubMed]

6. Cdc.gov. Reactions and Adverse Events of the Pfizer-BioNTech COVID-19 Vaccine j CDC. 2021. Available online: https: //www.cdc.gov/vaccines/covid-19/info-by-product/pfizer/reactogenicity.html (accessed on 4 February 2021).

7. Banerji, A.; Wickner, P.G.; Saff, R.; Stone, C.A., Jr.; Robinson, L.B.; Long, A.A.; Wolfson, A.R.; Williams, P.; Khan, D.A.; Phillips, E.; et al. mRNA vaccines to prevent COVID-19 disease and reported allergic reactions: Current evidence and suggested approach. J. Allergy Clin. Immunol. Pract. 2020, 9, 1423-1437. [CrossRef]

8. Wei, N.; Fishman, M.; Wattenberg, D.; Gordon, M.; Lebwohl, M. “COVID arm”: A reaction to the Moderna vaccine. JAAD Case Rep. 2021, 10, 92-95. [CrossRef] [PubMed]

9. Saifuddin, A.; Koesnoe, S.; Kurniati, N.; Sirait, S.; Arisanty, R.; Yunihastuti, E. COVID Arm After Moderna Booster in Healthcare Worker: A Case Report. Acta Med. Indones. 2021, 53, 326-330. [PubMed]

10. Johnston, M.S.; Galan, A.; Watsky, K.L.; Little, A.J. Delayed Localized Hypersensitivity Reactions to the Moderna COVID-19 Vaccine: A Case Series. JAMA Dermatol. 2021, 157, 716-720. [CrossRef]

11. Barriere, J.; Bondouy, M. COVID arm and PET/FDG imaging. Bull. Cancer 2021, 108, 668-669. [CrossRef]

12. Zengarini, C.; Artanidi, C.; Preci, C.; Gaspari, V. Erythema migrans-like rash after Moderna vaccine: An uncommon type of “COVID arm". Dermatol. Ther. 2021, 34, e15063. [CrossRef]

13. Kempf, W.; Kettelhack, N.; Kind, F.; Courvoisier, S.; Galambos, J.; Pfaltz, K. 'COVID arm'-Histological features of a delayed-type hypersensitivity reaction to Moderna mRNA-1273 SARS-CoV2 vaccine. J. Eur. Acad. Dermatol. Venereol. 2021, 35, e730-e732. [CrossRef] [PubMed]

14. Lindgren, A.L.; Austin, A.H.; Welsh, K.M. COVID Arm: Delayed Hypersensitivity Reactions to SARS-CoV-2 Vaccines Misdiagnosed as Cellulitis. J. Prim. Care Community Health 2021, 12, 21501327211024431. [CrossRef]

15. Mauriello, P.M.; Barde, S.H.; Georgitis, J.W.; Reisman, R.E. Natural history of large local reactions from stinging insects. J. Allergy Clin. Immunol. 1984, 74 (Pt 1), 494-498. [CrossRef]

16. Allergic Reactions. Centers for Disease Control and Prevention. Available online: https://www.cdc.gov/coronavirus/2019 -ncov/vaccines/safety/allergic-reaction.html (accessed on 26 August 2021).

17. Gambichler, T.; Boms, S.; Susok, L.; Dickel, H.; Finis, C.; Abu Rached, N.; Barras, M.; Stücker, M.; Kasakovski, D. Cutaneous findings following COVID-19 vaccination: Review of world literature and own experience. J. Eur. Acad. Dermatol. Venereol. 2022, 36, 172-180. [CrossRef]

18. Sun, Q.; Fathy, R.; McMahon, D.E.; Freeman, E.E. COVID-19 Vaccines and the Skin: The Landscape of Cutaneous Vaccine Reactions Worldwide. Dermatol. Clin. 2021, 39, 653-673. [CrossRef] 
19. Baden, L.R.; El Sahly, H.M.; Essink, B. Efficacy and safety of the mRNA-1273 SARS-CoV-2 vaccine. N. Engl. J. Med. 2020, 384, 403-416. [CrossRef]

20. Bennardo, L.; Nisticò, S.P.; Dastoli, S.; Provenzano, E.; Napolitano, M.; Silvestri, M.; Passante, M.; Patruno, C. Erythema Multiforme and COVID-19: What Do We Know? Medicina 2021, 57, 828. [CrossRef]

21. Dastoli, S.; Bennardo, L.; Patruno, C.; Nisticò, S.P. Are erythema multiforme and urticaria related to a better outcome of COVID-19? Dermatol. Ther. 2020, 33, e13681. [CrossRef] [PubMed]

22. Mazzitelli, M.; Dastoli, S.; Mignogna, C.; Bennardo, L.; Lio, E.; Pelle, M.C.; Trecarichi, E.M.; Pereira, B.I.; Nisticò, S.P.; Torti, C. Histopathology and immunophenotyping of late onset cutaneous manifestations of COVID-19 in elderly patients: Three case reports. World J. Clin Cases 2021, 9, 5744-5751. [CrossRef] [PubMed]

23. Khalid, M.; Lipka, O.; Becker, C. Moderna COVID-19 vaccine induced skin rash. Vis. J. Emerg. Med. 2021, 25, 101108. [CrossRef] [PubMed]

24. Ackerman, M.; Henry, D.; Finon, A.; Binois, R.; Esteve, E. Persistent maculopapular rash after the first dose of Pfizer-BioNTech COVID-19 vaccine. J. Eur. Acad. Dermatol. Venereol. 2021, 35, 423-425. [CrossRef] [PubMed]

25. Soyfer, V.; Gutfeld, O.; Shamai, S.; Schlocker, A.; Merimsky, O. COVID-19 vaccine-induced radiation recall phenomenon. Int. J. Radiat. Oncol. Biol. Phys. 2021, 110, 957-961. [CrossRef]

26. Munavalli, G.G.; Guthridge, R.; Knutsen-Larson, S. COVID-19/SARS-CoV-2 virus spike protein-related delayed inflammatory reaction to hyaluronic acid dermal fillers: A challenging clinical conundrum in diagnosis and treatment. Arch. Dermatol. Res. 2022, 314, 1-15. [CrossRef]

27. Lopatynsky-Reyes, E.Z.; Acosta-Lazo, H.; Ulloa-Gutierrez, R.; Ávila-Aguero, M.L.; Chacon-Cruz, E. BCG Scar Local Skin Inflammation as a Novel Reaction Following mRNA COVID-19 Vaccines in Two International Healthcare Workers. Cureus 2021, 13, e14453. [CrossRef] [PubMed]

28. Clinical Trial Data Moderna COVID-19 Vaccine (EUA). Available online: https:/ /www.modernatx.com/covid19vaccine-eua/ providers/clinical-trial-data (accessed on 4 February 2021).

29. McMahon, D.E.; Amerson, E.; Rosenbach, M.; Lipoff, J.B.; Moustafa, D.; Tyagi, A.; Desai, S.R.; French, L.E.; Lim, H.W.; Thiers, B.H.; et al. Cutaneous reactions reported after Moderna and Pfizer COVID-19 vaccination: A registry-based study of 414 cases. J. Am. Acad. Dermatol. 2021, 85, 46-55. [CrossRef]

30. Baden, L.R.; El Sahly, H.M.; Essink, B.; Kotloff, K.; Frey, S.; Novak, R.; Diemert, D.; Spector, S.A.; Rouphael, N.; Creech, C.B.; et al. Efficacy and safety of the mRNA-1273 SARS-CoV-2 vaccine. N. Engl. J. Med. 2020, 383, 2603-2615. [CrossRef] [PubMed]

31. De Montjove, L.; Marot, L.; Baeck, M. Eosinophilic cellulitis after BNT162b2 mRNA Covid-19 vaccine. J. Eur. Acad. Dermatol. Venereol. 2022, 36, e26-e28.

32. Yu, A.M.; Ito, S.; Leibson, T.; Lavi, S.; Fu, L.W.; Weinstein, M.; Skotnicki, S.M. Pediatric Wells syndrome (eosinophilic cellulitis) after vaccination: A case report and review of the literature. Pediatr. Dermatol. 2018, 35, e262-e264. [CrossRef]

33. Centers for Disease Control and Prevention. Local Reactions, Systemic Reactions, Adverse Events, and Serious Adverse Events: Moderna COVID-19 Vaccine. Available online: https:/ /www.cdc.gov/vaccines/covid-19/info-by-product/moderna/ reactogenicity.html (accessed on 12 February 2021). 